\title{
EFECTOS EN EL COEFICIENTE DE REFLEXIÓN DE UNA ANTENA DE MICROCINTA RECTANGULAR DEBIDOS A LAS MODIFICACIONES DE POSICIÓN DE UNA RANURA EN LA CAPA RADIANTE
}

\author{
OSCAR DAVID OSSA MOLINA ${ }^{1}$ \\ AIMEÉ LINETH FORERo CAMEN ${ }^{2}$ \\ CATHERINE ESPINAL RAMÍREZ ${ }^{3}$ \\ ERICK ESTEFEN REYES-VERA ${ }^{4}$ \\ FRANCISCO EUGENIO LÓPEZ GIRALDO ${ }^{5}$
}

\section{RESUMEN}

Este trabajo propone analizar los cambios en las frecuencias de resonancia de una antena de microcinta inducidos por una ranura grabada en la capa radiante, donde el objetivo es encontrar una relación clara entre los cambios en la frecuencia de resonancia debido a la posición y orientación de la ranura. Se utilizó un software numérico basado en Elementos Finitos para simular barridos paramétricos, lo que permitió identificar un patrón de cambio de la respuesta del coeficiente de reflexión de la antena. Los resultados muestran que las variaciones en la frecuencia de resonancia fundamental y los modos de resonancia de orden superior dependen de la orientación y posición de la ranura. La relación entre las frecuencias de resonancia y la posición y orientación de la ranura se describió por medio de modelos matemáticos, los cuales permiten sintonizar dichas frecuencias por medio de los parámetros geométricos de la ranura. Asimismo, se establece que los modos de resonancia $\left(T M^{*}\right)$ se afectan de acuerdo con la orientación de la ranura. Además, se presenta una alternativa de diseño de antenas multibanda.

PALABRAS CLAVES: Antenas de microcinta ranuradas, sintonización de frecuencia de resonancia, modelo línea de transmisión, cavidades resonantes, antenas de microcinta multibanda, coeficiente de reflexión S11.

1 Ingeniero de Telecomunicaciones, estudiante Maestría, Instituto Tecnológico Metropolitano.

2 Tecnólogo en Telecomunicaciones, estudiante Ingeniería, Instituto Tecnológico Metropolitano.

3 Tecnólogo en Telecomunicaciones, estudiante Ingeniería, Instituto Tecnológico Metropolitano.

4 Magíster en Ciencias Físicas. Docente ocasional tiempo completo, Instituto Tecnológico Metropolitano.

5 Doctor en Física. Docente de planta, Instituto Tecnológico Metropolitano.

Autor de correspondencia: Ossa Molina, O.D. (Oscar David): Instituto Tecnológico Metropolitano, Calle 54를 No. 30 01, Barrio Boston, Medellín, Colombia. C.P 050012. Correo electrónico: oscarossa@itm.edu.co
Historia del artículo:

Artículo recibido: 24-VII-2017/ Aprobado: 26-IV-2017

Disponible online: 30 de marzo de 2018

Discusión abierta hasta abril de 2019 


\title{
REFECTION COEFFICIENT EFFECTS ON MICROSTRIP PATCH ANTENNA DUE TO THE CHANGES OF POSITION OF SLOT PLACED ON ITS RADIATING PATCH
}

\begin{abstract}
This work proposes to analyze changes in the resonance frequencies of patch antenna induced by a slot on the radiant coat, where to find a clear relationship between changes in resonance frequency due to the position and orientation of the slot is the objective. We used a numerical software based on Finite Element method in order to simulate parametric sweeps of slot geometry, then it allowed us to identify a pattern of change in the response of the coefficient of reflection (S11) of the antenna. The results showed that variations in the fundamental resonance frequency and the resonance of higher-order modes depend on the orientation and position of the slot. The relationship between the resonance frequencies and the position and orientation of the slot was described by means of mathematical models, which allow to tune frequencies by means of geometric parameters of the slot such orientation and position. Also, it established that the modes of resonance $\left(T M^{*}\right)$ are affected according to the orientation of the slot. In addition, it is an alternative to multi-band patch antennas design.
\end{abstract}

KEYWORDS: Slotted patch antennas, resonance frequency tuning, Transmission Lines model, Cavity model, multiband patch antenna, reflection coefficient S11.

\section{EFEITOS NO COEFICIENTE DE REFLEXÃO DE UMA ANTENA DE MICROCINTA RETANGULAR DEVIDOS ÀS MODIFICAÇÕES DE POSIÇÃO DE UMA RANHURA NA CAPA RADIANTE}

\section{RESUMO}

Este trabalho propõe-se analisar as alterações nas frequências de ressonância de uma antena de microstrip induzida por uma ranhura no casaco radiante, o objetivo é encontrar uma clara relação entre mudanças na frequência de ressonância devido a posição e orientação da ranhura. Nós usamos um número baseado em software de elementos finitos para simular varreduras paramétricas, isto permitiu identificar um padrão de mudança da resposta do coeficiente de reflexão da antena. Os resultados mostram que as variações na frequência de ressonância fundamental e os modos de ressonância de ordem superior dependem da orientação e posição da ranhura. A relação entre as frequências de ressonância e a posição e orientação da ranhura descreveu-se por médio de modelos matemáticos, os quais permitiram sintonizar ditas frequências através dos parâmetros geométricos da ranhura. Assim mesmo, estabeleceu-se que os modos de ressonância $\left(T M^{*}\right)$ se afetam de acordo com a orientação da ranhura.

PALAVRAS-CHAVE: Antenas de microtira com fenda, sintonização de frequência de ressonância, modelo de linha de transmissão, cavidades ressonantes, antenas microstrip multibanda, coeficiente de reflexão S11. 


\section{INTRODUCCIÓN}

Las modificaciones del diseño de las antenas de microcinta son estudiadas con el objetivo de mejorar sus características de funcionamiento. Entre las modificaciones que se encuentra en la literatura, la adición de ranuras en el parche radiante de la antena permite obtener mejor directividad del haz de radiación (Kim et al. 2011), reducción de tamaño de la antena (Raval et al. 2015) y mayor ancho de banda de impedancia. Una característica que se desea, por medio de la adición de ranuras en las antenas de microcinta, es tener varias frecuencias de operación o resonancia multiband, lo que habilita a la antena funcionar, con un buen desempeño, en más de una frecuencia. El control de la frecuencia de resonancia de una antena tipo parche de geometría circular fue propuesto mediante el cambio de orientación de un arreglo de ranuras en la capa radiante de la antena (Reddy et al. 2015); los resultados allí encontrados muestran que para las frecuencias de orden superior es más notable el cambio debido a la orientación del arreglo de ranuras. En (Khidre et al. 2015) los autores proponen una antena de microcinta multiband mediante la adición de una ranura rectangular con un capacitor en el medio de esta, ambos (ranura y capacitor) posicionados en el centro de la antena; en los resultados, los autores encontraron que, la primera y segunda frecuencia de resonancia, las cuales pertenecen al modo fundamental, tienen relación con la capacitancia en la ranura; sin embargo, algunos de los requerimientos de pérdidas por retornos no cumplen con la restricción de - $10 \mathrm{~dB}$, aun así, es viable la configuración de una antena de dos bandas de operación mediante el uso de la técnica propuesta en ese trabajo. En lo referente a antenas de ultra ancho de banda (UWB, de sus siglas en ingles), se utilizó ranuras en una antena de microcinta de parche rectangular para lograr un perfecto rechazo de la banda de $5 \mathrm{Ghz}$, con la garantía de conservar las característica de UWB (Jang \& Hwang 2009). Otra investigación indaga sobre una alternativa de mejora de las desventajas intrínsecas de las antenas de microcinta, tales como baja ganancia y estrecha banda de operación (Khan et al. 2017). Los autores proponen la adición de ranuras en la capa radiante de la antena y, en los resultados, se encontró una ganancia de $8.5 d B i$ y $15.14 \%$ de ancho de banda para la banda $K a$, y una $11.84 d B i$ y $17.7 \%$ para la banda $K u$. Por otro lado y en relación de las antenas de microcinta con el campo de la metrología, se han propuesto diferente clases de sensores, entre estos: sensor de temperatura (Yang et al. 2012) (Fu \& Yang 2015), sensores de deformación (Yi et al. 2013) y sensores de identificación por radiofrecuencia (RFID, de sus siglas en inglés) (Calabrese \& Marrocco 2008).

Algunas investigaciones han indagado en la relación que podría existir entre la geometría de una modificación en el parche radiante de la antena de microcinta y el cambio en la frecuencia de resonancia de la misma. Por ejemplo, en (Joler \& Kucan 2015), los autores realizan la documentación de pruebas experimentales y mediante simulaciones de la variación en los parámetros de longitud, ancho y posición de una ranura rectangular ubicada en el parche de la antena. Este estudio se realizó para los tres primeros modos de resonancia. En los resultados, los autores enfatizan de forma cualitativa que cada frecuencia de resonancia tiene diferente susceptibilidad a cambios ante el efecto de la ranura, sin embargo, no se tiene una expresión matemática que relacione las características geométricas de la ranura y los cambios en las frecuencias de resonancia analizadas, y la discusión no responde a el por qué los modos de resonancia se afectan en forma diferente debido a una configuración especifica de la ranura. De forma similar en (Shivnarayan \& Vishvakarma 2005) se estudia como la impedancia de entrada varía cuando se agrega una ranura en la capa radiante de la antena de microcinta. Los resultados mostraron que la impedancia de entrada y la frecuencia de resonancia cambian a medida que el ancho de la ranura cambia, además, los datos que relacionan las dimensiones de la ranura y las características eléctricas de la antena podrían ajustarse a un comportamiento lineal; este experimento se 
realizó para diferentes valores fijos de longitud de la ranura, sin embargo, solo se analiza una orientación y posición. Por otro lado, para el diseño de antenas de microcinta se utiliza modelos empíricos como el de líneas de transmisión y cavidades resonantes, métodos que están limitados a geometrías regulares como la rectangular y circular. Para el caso de diseños más complejos se hace necesario el uso de software basado en métodos de solución de onda completa, como lo es el Método de Elementos Finitos (FEM), los cuales proporcionan mejores soluciones, pero requieren de un alto costo computacional y, dependiendo del número de factores que se evalué, este procedimiento podría tomar una cantidad de tiempo significativa. Recientemente, CatanoOchoa et.al. realizaron un análisis del comportamiento espectral de una antena microcinta cuando sobre el parche radiante era grabado un arreglo de resonadores hexagonales, los cuales presentaban un comportamiento metamaterial. Los resultados mostraron que para este caso particular la frecuencia de resonancia puede ser alterada si se modifica la distancia entre los resonadores y por ende la forma en cómo es distribuida la densidad de corriente sobre el parche (Catano-Ochoa et al. 2016).

El presente trabajo tiene como objetivo investigar la relación que existe entre la posición y orientación (sea vertical u horizontal) de una ranura rectangular ubicada en la capa radiante de una antena de microcinta rectangular, y los consecuentes cambios en la frecuencias de resonancia. Con ello, se busca obtener expresiones matemáticas que relacionen las frecuencias de resonancia de la antena con los parámetros geométricos de la ranura. Estas expresiones matemáticas brindarán una herramienta que permitirá obtener frecuencias específicas y sintonizar su comportamiento a través de la alteración de la posición y orientación de la ranura. Finalmente, se pretende contribuir en la construcción de modelos de análisis de antenas de microcinta, con el fin de prescindir, en trabajos futuros, de programas comerciales de análisis electromagnético basados en técnicas numéricas complejas (Método de momentos, método de elementos finitos, etc.).

\section{MATERIALES Y MÉTODOS}

\section{A. Influencia de una ranura rectan-} gular sobre la capa radiante de una antena de microcinta

Teniendo en cuenta que los diferentes diseños de antenas de microcinta varían en su desempeño, lo cual es evidente en los parámetros como el ancho de banda, patrón de radiación, frecuencia de resonancia; entonces existe la necesidad de encontrar alguna relación que describa las modificaciones en el diseño de la antena y la influencia de dichos cambios sobre sus características de funcionamiento. Una antena de microcinta rectangular puede modelarse como un circuito RLC resonante, donde los parámetros de inductancia, capacitancia y resistencia están determinados por las dimensiones de la antena (ancho $W$, largo $L$ y espesor $h$ ). Cuando se realiza una modificación en su diseño, tal como una ranura rectangular, la impedancia de entrada de la antena cambia en función de las dimensiones y la posición de la ranura (Shivnarayan \& Vishvakarma 2005). Lo anterior conduce a cambios en el coeficiente de reflexión de la antena (S11 de los Scattering Parameters), los cuales están descritos por la variación de la impedancia de entrada debido a la adición de la ranura en su capa radiante, tal como lo muestra la Ecuación 1.

$$
Z_{\text {in }}=\frac{(R-j X)\left(j X_{s}\right)}{R-j X+j X_{s}}
$$

Donde $X_{s}$ es la reactancia debida a la ranura adicionada y su valor depende de su geometría y posición. El coeficiente de reflexión relaciona la impedancia de la línea de transmisión (impedancia característica que depende del sistema) y la impedancia de la carga (antena) conectada al final de la línea. Cuando hay un acople de impedancia de los dos medios, se tiene máxima transferencia de la onda incidente hacia la antena, de lo contrario, dominan las reflexiones entre los dos medios. El coeficiente 
de reflexión está relacionado con la frecuencia de resonancia del sistema, pues en la expresión de la Ecuación 1 se nota que la parte compleja de la impedancia depende de la frecuencia a la cual se está caracterizando el sistema. Por lo tanto, existe una relación entre las dimensiones ( $\mathrm{L}$ y $\mathrm{W}$ ) de la ranura con la impedancia característica de la antena. A su vez, la impedancia característica está relacionada con la frecuencia de resonancia de la antena, la cual es de interés para este caso de estudio.

\section{B. Diseño de la antena}

Para el análisis de los efectos de la ranura en la frecuencia de resonancia de la antena, se propone dos diseños de antenas de microcinta rectangular. El primero de estos consta de una antena (a la que se hará referencia como Antena 1) que opera a una frecuencia fundamental $f_{010}=2.9 \mathrm{GHz}$ y es diseñada sobre el sustrato dieléctrico RT/Duroid 6010 LM; el cual tiene una constante dieléctrica $\varepsilon_{r}=10.2$ y una altura $h=1.27 \mathrm{~mm}$. La estructura de esta antena es ilustrada en la Figura 1(A). Por otra parte, la segunda antena (Antena 2), es considerablemente más grande en relación a la primera como se puede observar en la Figura 1(B). Esta es diseñada para una frecuencia fundamental $f_{010}=2.3 \mathrm{GHz}$ y utiliza un sustrato dieléctrico de fibra de vidrio (FR-4) con una constante dieléctrica $\varepsilon_{r}=4.3$ y una altura $h=1.6$ $\mathrm{mm}$. Las dos antenas fueron diseñadas siguiendo los lineamientos del modelo de análisis de líneas de transmisión (Balanis 2005), el cual refiere el diseño de la antena para el modo de resonancia dominante, es decir, la frecuencia de resonancia de menor valor que se determinada por la longitud $L$ del parche radiante. La secuencia de ecuaciones 2-5 describe el procedimiento de diseño las antenas, allí se requiere del conocimiento previo de la constante dieléctrica $\varepsilon_{r}$ y $h$ altura del sustrato dieléctrico usado, y la frecuencia de resonancia para la cual se diseñará la antena.

$$
W=\frac{1}{2 \mathrm{f}_{\mathrm{r}} \sqrt{\mu_{0} \varepsilon_{0}}} \sqrt{\frac{2}{\varepsilon_{\mathrm{r}}+1}}
$$

$$
\begin{gathered}
\varepsilon_{\text {reff }}=\frac{\varepsilon_{\mathrm{r}}+1}{2}+\frac{\varepsilon_{\mathrm{r}}-1}{2}\left(1+12 \frac{\mathrm{h}}{\mathrm{W}}\right)^{\frac{-1}{2}} \\
\Delta \mathrm{L}=0.412 \mathrm{~h} \frac{\left(\varepsilon_{\text {reff }}+0.3\right)\left(\frac{\mathrm{w}}{\mathrm{h}}+0.264\right)}{\left(\varepsilon_{\text {reff }}-0.258\right)\left(\frac{\mathrm{w}}{\mathrm{h}}+0.8\right)} \\
\mathrm{L}=\frac{1}{2 \mathrm{f}_{\mathrm{r}} \sqrt{\varepsilon_{\text {reff }}} \sqrt{\mu_{0} \varepsilon_{0}}}-2 \Delta \mathrm{L}
\end{gathered}
$$

Donde $\frac{1}{\sqrt{\mu_{0} \varepsilon_{0}}}$ es la velocidad de la luz, $\Delta L$ es la extensión de la longitud de la capa radiante debido al efecto de borde (fringing effect), $W$ y $L$ y es el ancho y largo del parche respectivamente. Antena $1 \mathrm{y}$ Antena 2 se modificaron mediante la adición de una ranura de $0.5 \mathrm{~mm}$ y $1 \mathrm{~mm}$ espesor, respectivamente; con un ancho y una longitud de $2 \mathrm{~mm}$ menor que la dimensión del borde ( $W$ y $L$ para la orientación horizontal y vertical respectivamente), tal como se observar en la Figura 1 (A) y Figura 1 (B). Los diseños esquemáticos de las antenas propuestas y sus respectivas dimensiones se muestran de forma detallada en la Figura 1.

Figura 1. Diseño y medidas geométricas de las antenas de microcinta rectangular con ranuras. A) Antena 1 y B) Antena 2. Todas las medidas en milímetros [ $\mathrm{mm}]$.

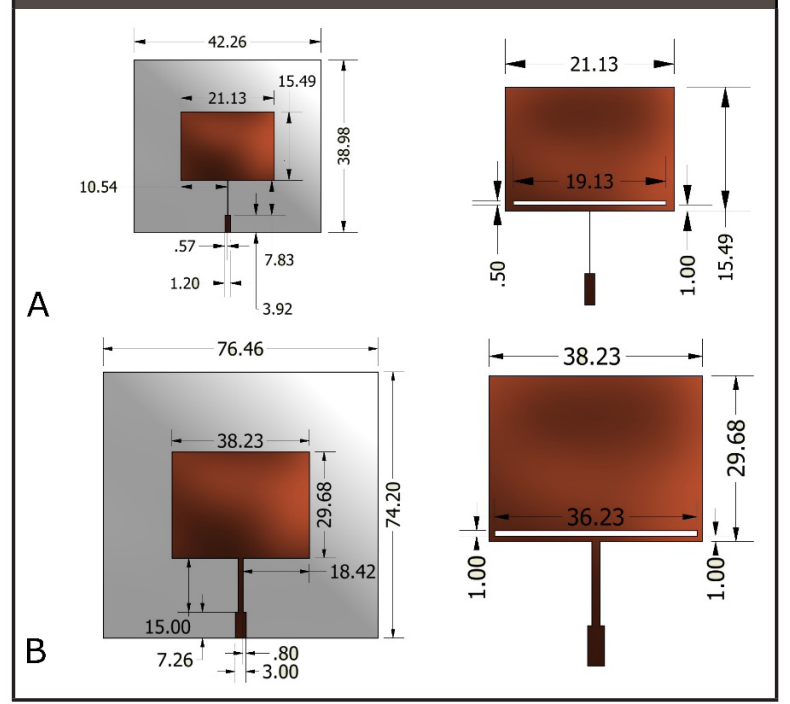




\section{Simulaciones paramétricas}

Para llevar a cabo el análisis computacional de las antenas diseñadas, se empleó el software comercial Computer Simulation Technology (CST). En esta etapa del trabajo se realizó un análisis con el fin de obtener el coeficiente de reflexión (S11) asociado a cada una de las antenas propuestas. Los resultados obtenidos muestran coincidencia con las frecuencias fundamentales calculadas en la etapa de diseño de las antenas, y aparecieron nuevos puntos de resonancia de orden superior (otros modos de resonancia), los cuales son de considerar para el análisis. En Figura 2 se muestra el coeficiente de reflexión de las dos antenas sin la ranura y con la ranura con orientación vertical y horizontal. Antes de agregar la ranura, mostrado en Figura 2. a) y d), las frecuencias de resonancia de las antenas corresponden al diseño sin ninguna modificación, donde las frecuencias de resonancia de menor valor hacen referencia al modo de resonancia fundamental de cada antena. Cuando es grabada una ranura orientada verticalmente, es decir, paralela a la línea de alimentación, se presenta una perturbación en el parámetro $S 11$, tal como se muestra en Figura 2. b) y e). En consecuencia de este cambio, se genera la aparición de nuevas frecuencias de operación en cada antena, en las frecuencias de 3.7 y $5 \mathrm{GHz}$ para la antena 1 , y 3.05 y $4.5 \mathrm{GHz}$ para la antena 2. Adicionalmente, se aprecia que la frecuencia de resonancia fundamental permanece casi constante para ambas antenas.

Figura 2. Coeficiente de reflexión de Antena 1 y Antena 2. A) y D) frecuencias de resonancia sin ranuras o antena sin ninguna modificación, B) y E) cambio en el S11 y nuevas frecuencias de resonancia debido a la adición de la ranura en orientación vertical, y C) y F) cambio en el S11 y nuevas frecuencias de resonancia debido a la ranura en orientación horizontal.

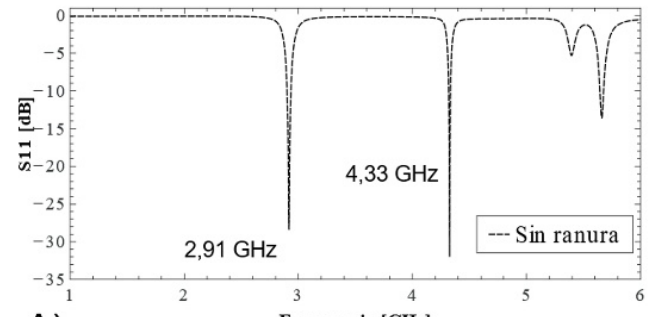

A)
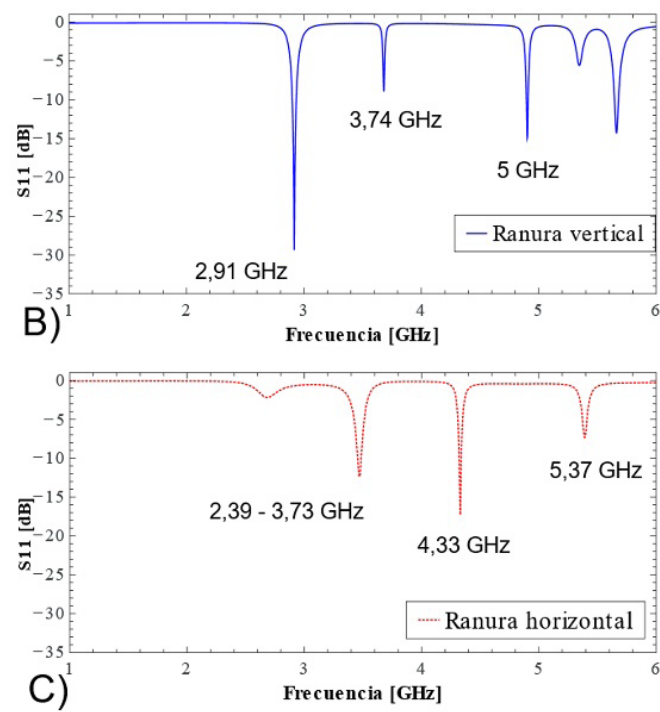

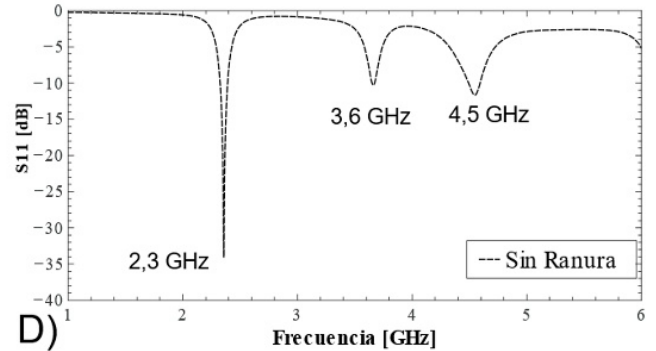

D) Frecuencia $[\mathrm{GHz}]$
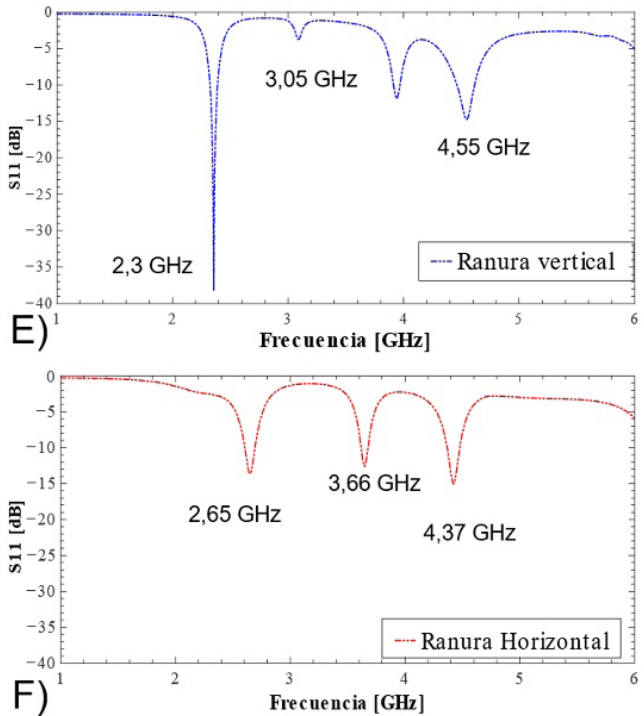
Por otro lado, al grabar la ranura con una orientación horizontal (ortogonal a la línea de alimentación), también existe una perturbación en el parámetro $S 11$, como se muestra en Figura 2. c) y f), lo que resulta en nuevas frecuencias de resonancia alrededor de 2.3, 3.7 y $5.37 \mathrm{GHz}$ para la antena 1 , y 2.6 y 4.3 $\mathrm{GHz}$ para la antena 2. En esta ocasión, las frecuencias fundamentales de cada antena se ve mucho más perturbadas en comparación con la ranura en posición vertical, y por el contrario, las frecuencias de resonancia de orden superior, específicamente 4.3 y 3.6 $\mathrm{GHz}$ para Antena 1 y Antena 2, respectivamente, no sufren modificaciones notables. Con el objetivo de evidenciar cómo cambia el parámetro $S 11$ de las antenas debido a la ranura con orientación vertical y horizontal, se propone una simulación variando la posición de la ranura sobre el largo y ancho del parche de la antena. Dicho análisis se realiza de forma independiente con el fin de comprender mejor los resultados obtenidos y correlacionar las variaciones en las frecuencias de resonancia debido al desplazamiento de la ranura en cada dirección.

Una vez se pudo determinar que al incluir una ranura sobre el parche radiante de las antenas de mi- crocinta se puede generar otras frecuencias de resonancia, el siguiente paso es llevar a cabo un análisis de la posición de la ranura y las modificaciones del parámetro S11. La ranura recorrerá, tanto en orientación vertical como en orientación horizontal, toda el área del parche radiante de la antena. En Figura 3 se describe el proceso de simulación propuesto. Primero, para ambas antenas, la ranura fue puesta en orientación horizontal a $1 \mathrm{~mm}$ de separación desde el borde inferior del parche radiante (borde donde se conecta la línea de alimentación). En adelante, la posición de la ranura aumento en pasos de $d_{y}\left(d_{y}=\right.$ $0.5 \mathrm{~mm}$ y $d_{y}=1 \mathrm{~mm}$, respectivamente para Antena 1 y Antena 2) hasta llegar al borde del otro extremo. Después, las ranuras fueron puestas en orientación vertical a un distancia de $1 \mathrm{~mm}$ desde el borde más a la izquierda del parche de la antena, el aumento de la posición de la ranura $\left(d_{y}\right)$ conservó su valor para cada antena. Las ranuras fueron grabadas con un espesor de $0.5 \mathrm{~mm}$ y $1 \mathrm{~mm}$ para Antena 1 y Antena 2, respectivamente, además, los análisis de se realizaron de forma independiente para cada orientación de la ranura.

Figura 3. Configuración de la simulación paramétrica propuesta para Antena 1 y Antena 2. Las simulaciones se realizaron de forma independiente para cada una de las orientaciones de la ranura.

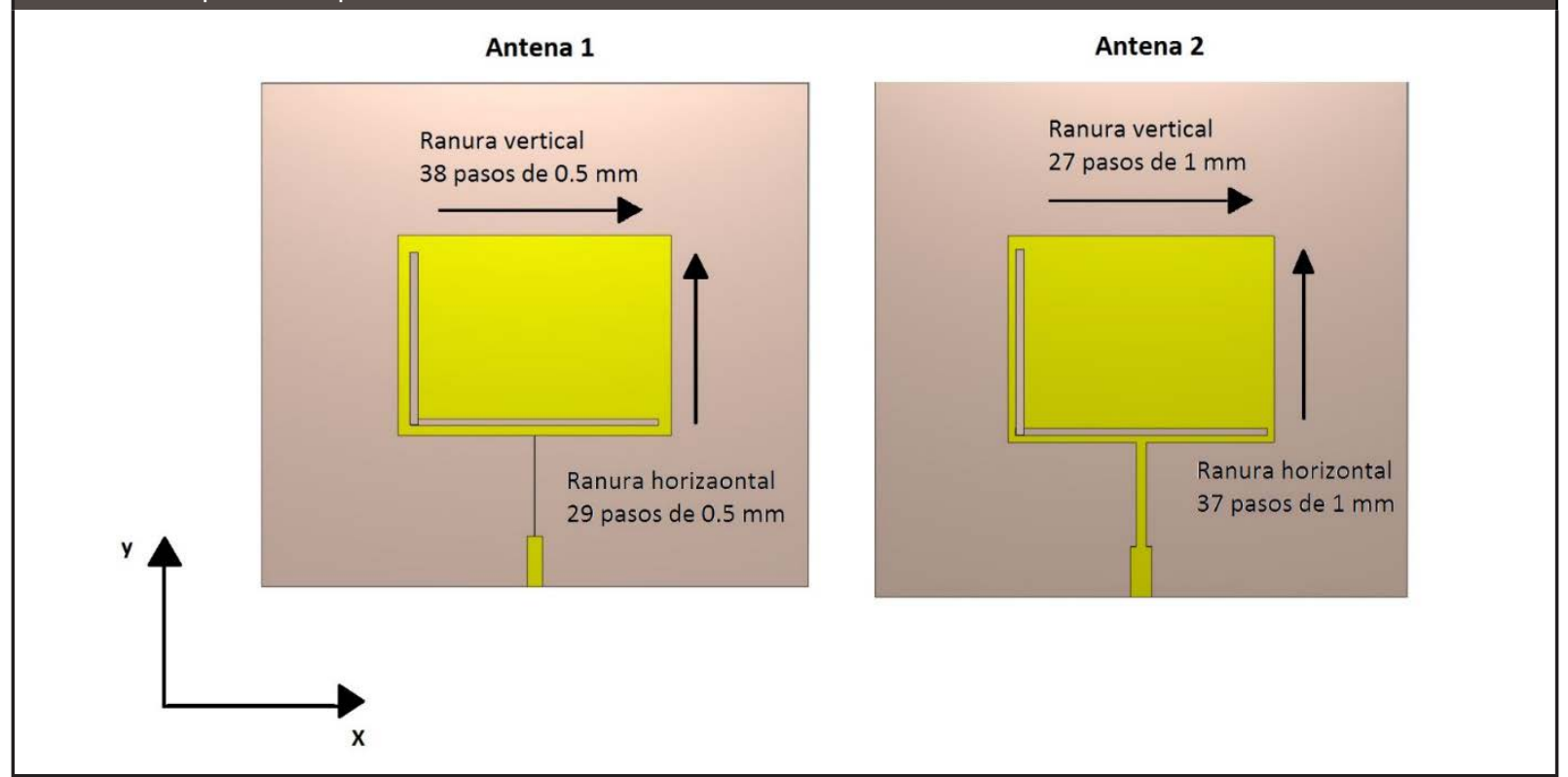




\section{RESULTADOS}

Las modificaciones en el parámetro $S 11$ para cada una de las antenas fueron estudiadas. Se realizó un seguimiento de las frecuencias de resonancia de cada antena con el objetivo de encontrar una expresión matemática que diera cuenta de las variaciones en las frecuencias de resonancia en función de la posición y orientación de la ranura sobre su capa radiante. En Figura 4 se muestra los resultados del cambio en el coeficiente de reflexión de la antena a medida que la ranura en orientación horizontal recorre la capa radiante de esta. De los resultados obtenidos, es evidente que las frecuencias de resonancia varían en función de la posición de la ranura. Donde dichas variaciones se representan por segmentos, lo cual se debe a que para algunas posiciones de la ranura no hay datos de frecuencia de resonancia asociados, tal como se muestra para Antena 1 en las frecuencias de resonancia $2.39 \mathrm{y}$ $5.37 \mathrm{GHz}$ y para Antena 2 en $2.65 \mathrm{GHz}$. También, las variaciones en la frecuencia de resonancia debido a la posición de la ranura se presentaron de forma continua, es decir, hubo valores de frecuencia para todo el rango de posiciones de la ranura, tal como se muestra para Antena 2 en la frecuencia de resonancia 4.37 y $1.57 \mathrm{GHz}$, de las cuales la última no fue referenciada en un principio (en Figura 2) pero que es de interés para el análisis debido a la tendencia de sus variaciones en relación la posición de la ranura. En el mismo sentido, en Figura 5 se muestra las modificaciones en el coeficiente de reflexión de las antenas, en este caso, la ranura tiene una orientación vertical y, para Antena 1 y Antena 2 , se puede hacer un seguimiento continuo de las frecuencias de resonancia en 3.74 y $3.05 \mathrm{GHz}$ respectivamente. Además, para la antena 1 en $5 \mathrm{GHz}$ el seguimiento de la frecuencia de resonancia resulto en 5 segmentos. Los trazos rojos que aparecen las Figuras 4 y 5 corresponden un ajuste de curva polinómica entre las variables frecuencia de resonancia (en $\mathrm{GHz}$ ) y posición de la ranura (en $\mathrm{mm}$ ).

Figura 4. Cambios en el coeficiente de reflexión debido a la variación de posición de la ranura en orientación horizontal. Antena 1: A) frecuencia de resonancia de 2.39 - $3.73 \mathrm{GHz}$ y B) $5.37 \mathrm{GHz}$. Antena 2: C) frecuencia de resonancia de $2.65 \mathrm{GHz}$, D) $4.37 \mathrm{GHz}$ y E) nueva frecuencia en $1.57 \mathrm{GHz}$

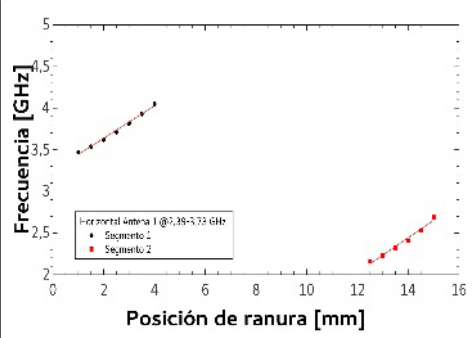

A)

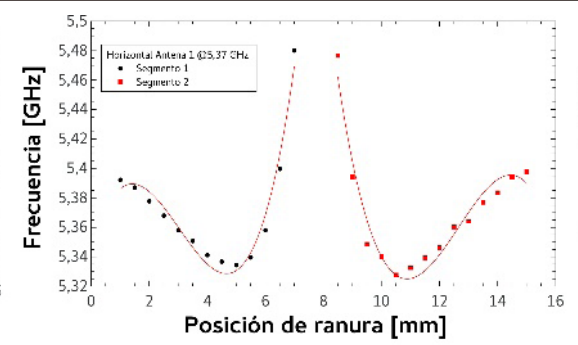

B)

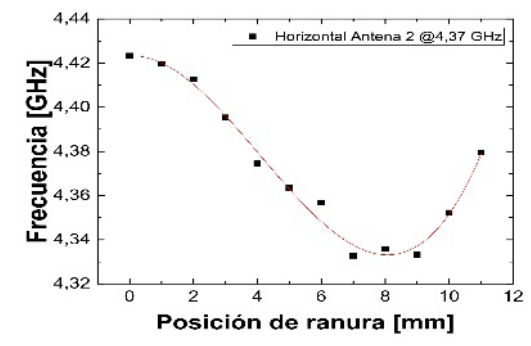

D)

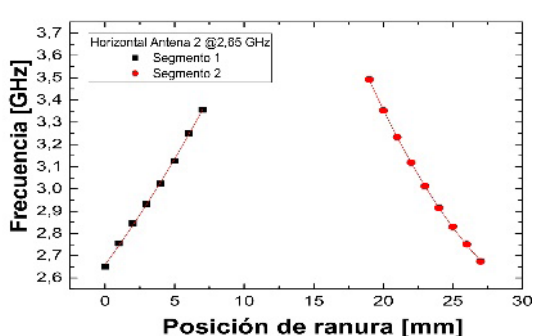

C)

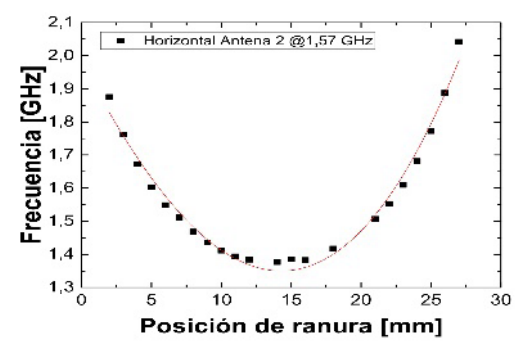

E) 
Figura 5. Cambios en el coeficiente de reflexión debido a la variación de la posición de la ranura en orientación vertical. Antena 1: A) frecuencia de resonancia de $3.74 \mathrm{GHz}$ y B) $6 \mathrm{GHz}$. Antena 2 C) frecuencia de resonancia de $3.05 \mathrm{GHz}$.

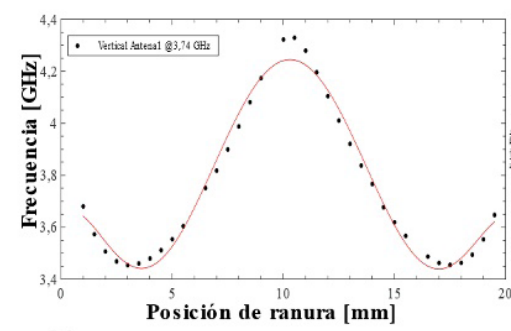

A)

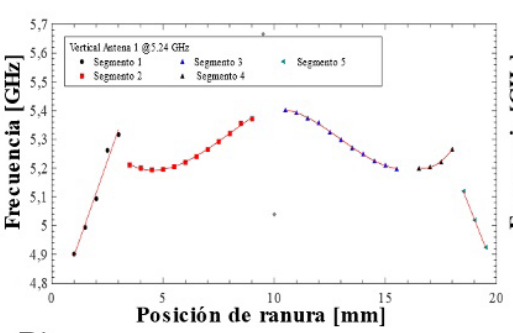

B)

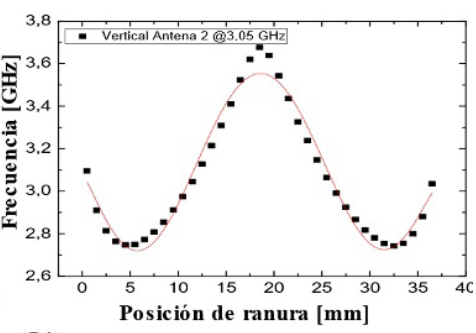

C)
En Tabla 1 y Tabla 2 se recopila los modelos matemáticos resultantes del ajuste realizado los datos analizados en las simulaciones paramétricas, mostrados en Figura 4 y Figura 5. Para todos los ajustes realizados se especifica la antena y orienta- ción de la ranura, el coeficiente $R^{2}$, el cual determina la calidad del modelo de predicción; y, finalmente, las expresiones matemáticas que describen el modelo, esto para cada uno de los segmentos analizados en las respectivas frecuencias de resonancia.

TABLA 1. AJUSTE DE CURVAS POLINÓMICAS DE LAS VARIACIONES EN LAS FRECUENCIAS DE RESONANCIA DE ANTENA 1 Y ANTENA 2 DEBIDO A LA POSICIÓN DE LA RANURA EN ORIENTACIÓN HORIZONTAL

Antenas posición Horizontal

\begin{tabular}{c|c|c|c}
\hline Nombre & Segmento & $\boldsymbol{R}^{\mathbf{2}}$ & Ecuación \\
\hline \multirow{2}{*}{ Antena 1 frecuencia @ 2,39-3,73 GHz } & 1 & $R^{2}=0,991$ & $Y=0,1943 X+3,2486$ \\
\cline { 2 - 4 } & 2 & $R^{2}=0,978$ & $Y=0,2086 X-0,4776$ \\
\hline \multirow{2}{*}{ Antena 1 frecuencia @ 5,37 GHz } & 1 & $R^{2}=0,968$ & $Y=0,0036 X^{3}-0,0326 X^{2}+0,0711 X+5,3441$ \\
\cline { 2 - 4 } & 2 & $R^{2}=0,949$ & $Y=-0,0031 X^{3}+0,1183 X^{2}-1,4694 X+11,324$ \\
\hline \multirow{2}{*}{ Antena 2 frecuencia @ 2,65 GHz } & 1 & $R^{2}=0,997$ & $Y=0,0993 X+2,6446$ \\
\cline { 2 - 4 } & 2 & $R^{2}=0,990$ & $Y=-0,1015 X+5,3756$ \\
\hline Antena 2 frecuencia @ 4,37 GHz & 1 & $R^{2}=0,987$ & $Y=0,0004 X^{3}-0,0044 X^{2}+0,0009 X+4,4232$ \\
\hline Antena 2 frecuencia @ 1,57 GHz & 1 & $R^{2}=0,986$ & $Y=3 \times 10^{-5} X^{3}+0,0022 X^{2}-0,0823 X+1,9829$ \\
\hline
\end{tabular}

TABLA 2. AJUSTE DE CURVAS POLINÓMICAS DE LAS VARIACIONES EN LAS FRECUENCIAS DE RESONANCIA DE ANTENA 1 Y ANTENA 2 DEBIDO A LA POSICIÓN DE LA RANURA EN ORIENTACIÓN VERTICAL

Antenas posición Vertical

\begin{tabular}{c|c|c|c}
\hline Nombre & Segmento & $\boldsymbol{R}^{\mathbf{2}}$ & Ecuación \\
\hline Antena 1 frecuencia @ 3,74 GHz & 1 & $R^{2}=0,956$ & $Y=0,0003 X^{4}-0,0118 X^{3}+0,1524 X^{2}-0,6335 X+4,2303$ \\
\hline \multirow{3}{*}{ Antena 1 frecuencia @ 5 GHz } & 1 & $R^{2}=0,981$ & $Y=0,2194 X+4,6754$ \\
\cline { 2 - 4 } & 2 & $R^{2}=0,988$ & $Y=0,0084 X^{2}-0,0716 X+5,3518$ \\
\cline { 2 - 4 } & 3 & $R^{2}=0,989$ & $Y=0,0001 X^{2}-0,0486 X+5,9078$ \\
\cline { 2 - 4 } & 5 & $R^{2}=0,996$ & $Y=0,0396 X^{2}-1,3232 X+16,253$ \\
\hline \multirow{2}{*}{ Antena 2 frecuencia @ 3,05 GHz } & 1 & $R^{2}=0,999$ & $Y=-0,1951 X+8,7291$ \\
\hline
\end{tabular}


En Figura 6 y Figura 7 se muestran el análisis del seguimiento de las frecuencias de resonancia de las antenas que presentaron un comportamiento diferente, que se consideró no relevante para el objetivo de sintonización de la frecuencia de resonancia por medio la posición y orientación de la ranura. Aunque existe una modificación en el parámetro $\mathrm{S} 11$, los cambios en la frecuencia de resonancia debido a la posición de la ranura muestra una dispersión de puntos sin una tendencia, o con variaciones muy pequeñas en comparación con los resultados mostrados en Figura 4 y Figura 5. Por lo tanto se considera que de estos resultados no se permite una sintonización de la frecuencia de resonancia en el rango de los $\mathrm{GHz}$ (para aplicaciones de microondas), sin embargo, un análisis en radiofrecuencias alrededor de $\mathrm{KHz}$ podría mostrar una tendencia interesante para estos resultados.

\section{DISCUSIÓN}

Al comparar el coeficiente de reflexión o parámetro S11 de las antenas antes y después de adicionar la ranura en la primera posición, tanto para la orientación horizontal como para la vertical, es notable que debido a la ranura se presentan nuevas frecuencias de resonancia, lo habilita a la antena a funcionar simultáneamente en varias frecuencias o aplicaciones. Es evidente que la respuesta del coeficiente de reflexión de cada antena es muy diferente de acuerdo con la orientación de la ranura adicionada. En efecto, en los resultados se puede ver que para las antenas con la ranura en orientación vertical, las frecuencias de resonancia fundamentales $f_{010}=2.91$ y $f_{010}=3.6 \mathrm{GHz}$ para Antena 1 y Antena 2, respectivamente, no sufren variaciones significativas; por el contrario, las frecuencias de resonancia de orden superior (resonancia debido a $W f_{002}$ ) se ven altamente alteradas debido a esta orientación de la ranura.

\section{Figura 6. Cambios en el coeficiente de reflexión debido a la variación de la posición de la ranura en orientación horizontal.} Antena 1: A) frecuencia en $4.33 \mathrm{GHz}$. Antena 2: B) frecuencia en $3.66 \mathrm{GHz}$

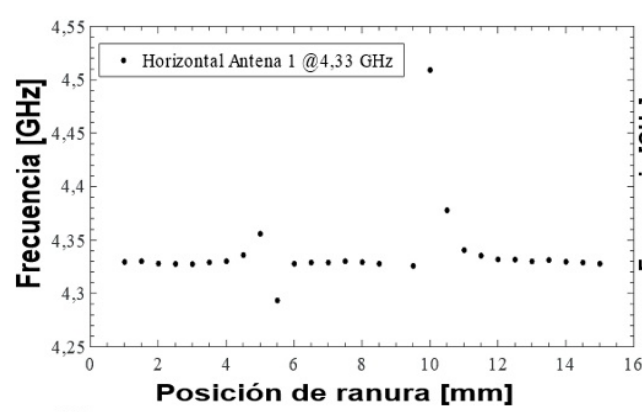

A)

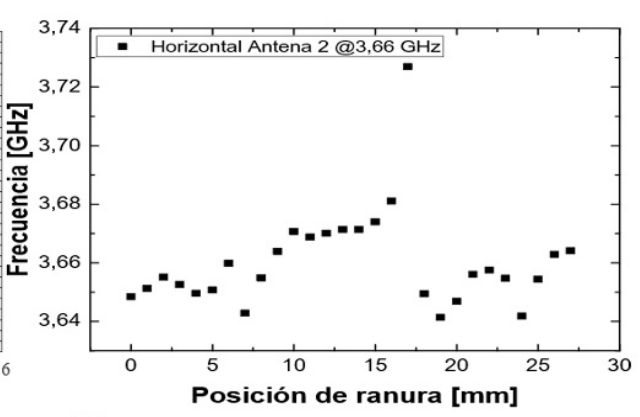

B)

Figura 7. Cambios en el coeficiente de reflexión debido a la variación de la posición de la ranura en orientación vertical. Antena 1: A) frecuencia en $2.91 \mathrm{GHz}$. Antena 2: frecuencia en B) $3.66 \mathrm{GHz}, \mathrm{C}) 4.55 \mathrm{GHz}$

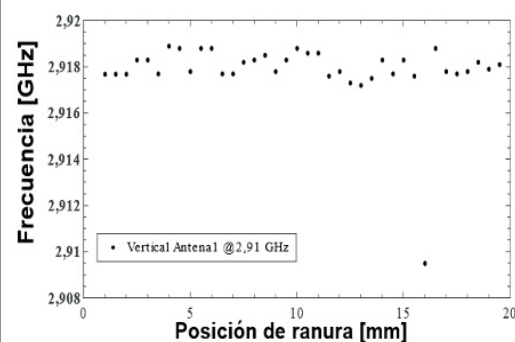

A)

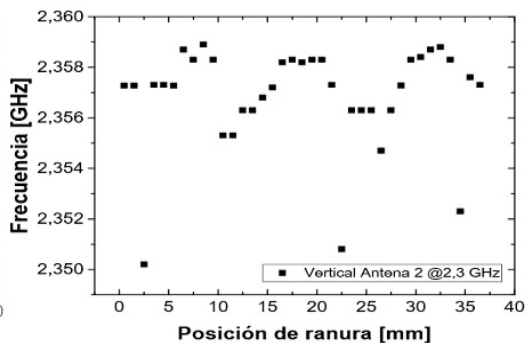

B)

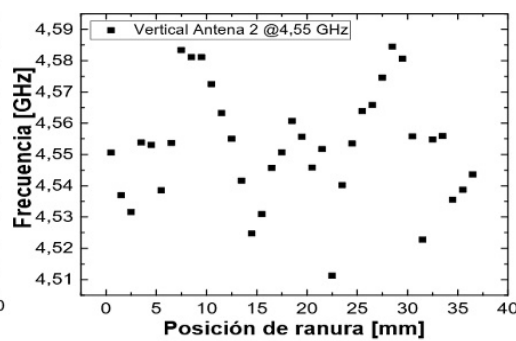

C) 
En contraste, cuando la ranura es orientada horizontalmente, las frecuencias $f_{002}=4.33$ y $f_{002}=$ 3.6 GHz para Antena 1 y Antena 2 respectivamente, se ven ligeramente afectadas debido a la presencia de la ranura, mientas que, las frecuencias de resonancia fundamentales para las dos antenas, fueron altamente afectadas dando lugar a otras frecuencias de resonancia. Una explicación teórica de los cambios en las frecuencia de resonancia de acuerdo a la orientación de la ranura se puede inferir al revisar el modelo de análisis de cavidades resonantes (Balanis 2005). Este modelo establece que el modo dominante (frecuencia de resonancia fundamental) está determinado por la longitud $L$ del parche radiante de la antena y el siguiente modo de orden superior (segundo modo de resonancia y sus harmónicos) está determinado por la dimensión del ancho $W$ del parche de la antena. Por lo anterior, al orientar la ranura perpendicular a la línea de alimentación (orientación horizontal) la distribución de la densidad de corriente para la frecuencia fundamental se verá obstaculizada por dicha ranura, generando un aumento en la longitud L del parche (perceptible eléctricamente) que conduce a una disminución en esta frecuencia de resonancia debido a la relación inversa que tiene $L$ con la misma; bajo el mismo argumento, al orientar la ranura de forma vertical, la distribución de la densidad de corriente para el modo de resonancia de orden superior $f_{002}$ (determinado por el parámetro $W$ ) se ve obstaculizada por la ranura y la frecuencia de resonancia tiende también a valores inferiores. Entonces, la variación de la frecuencia de resonancia está estrechamente relacionada con la distribución de corriente y el diseño del parche radiante de la antena. Para el caso aquí estudiado, cuando la ranura está en orientación vertical no se presenta una variación significativa en la longitud eléctrica $L$ del parche y la frecuencia de resonancia fundamental $\left(f_{010}\right)$ se afecta muy poco debido a que la ranura representa un obstáculo pequeño para la distribución de densidad de corriente en dicha frecuencia; mientras que la frecuencia de resonancia de orden superior $\left(f_{002}\right)$ y los otros mo- dos de resonancia son más susceptibles a cambios y se permiten sintonizar de acuerdo la posición de la ranura en dicha orientación. Por otro lado, cuando la ranura es puesta en orientación horizontal, la frecuencia de segundo $f_{002}$ se afecta levemente, dando lugar a la sintonización de la frecuencia de resonancia fundamental y otras de orden superior, por medio de la posición de la ranura. El argumento anterior se puede entender gráficamente al revisar en Figura 6 y Figura 7, donde se aprecia que las frecuencias de resonancia $f_{010}$ y $f_{002}$ se ven muy poco modificadas debido a la orientación vertical y horizontal de la ranura, respectivamente.

Los datos consignados en Tabla 1 y Tabla 2 muestran que los ajustes de curva polinómica tienen un coeficiente de determinación $R^{2}$ próximo 1 , en el mayor de los casos, y a su vez, hace entender que al utilizar dicho modelo para predecir una frecuencia de resonancia de acuerdo a la posición y orientación de la ranura, el resultado obtendrá un error considerablemente pequeño. Vale la pena aclara que los modelos aquí presentados tienen dicho comportamiento en el intervalo de la posición de la ranura estudiado, lo que conduce a establecer que para otras diseños de antenas de microcinta, se requiere de una nueva caracterización del coeficiente de reflexión para obtener las curvas que relacionan la frecuencia de resonancia con los parámetros geométricos de la ranura.

Desde el punto de vista de la sintonización de la frecuencia de resonancia o la búsqueda de un modelo matemático que relacione los datos de frecuencia de resonancia con los parámetros geométricos de la ranura sobre la antena, los resultados mostrados en Figura 6 y Figura 7 presentan variaciones muy pequeñas (alrededor de los $\mathrm{KHz}$ ) que en la escala de las microondas se podrían considerar como una frecuencia constante; o variaciones que no presentan una tendencia que se podría modelar por medio de una curva polinómica. Finalmente, en Figura 6 b) y Figura 7 c), las dispersiones de los puntos toman valores que son difícil de determinar con buena precisión utilizando un modelo de ajuste 
de curva polinómica, como se hizo para la distribución de datos en Figura 4 y Figura 5, pero los cuales podrían ser modelados con otro tipo de funciones, tales como las funciones periódicas.

\section{CONCLUSIONES}

Este trabajo mostro que existe una relación entre las modificaciones en el diseño de una antena de microcinta, tal como la adición de una ranura en su capa radiante, y sus características de funcionamiento como el confidente de reflexión y las frecuencias de resonancia. Asimismo se presentó modelos matemáticos que relacionan la frecuencia de resonancia con los parámetros de orientación y posición de la ranura, para dos antenas de microcinta de parche rectangular. Estos modelos hacen viable la sintonización de las frecuencias de resonancia en función los parámetros geométricos de la ranura sobre la capa radiante de la antena.

El análisis propuesto resulta en un método para la determinación de la frecuencia de resonancia de la antena por medio de la posición y orientación de la ranura, lo que permite cambiar las frecuencias de operación o generar antenas multibanda, de acuerdo con la orientación y posición de la ranura.

Los modos de resonancia se condicionan a las dimensiones y diseño de la antena, y estos determinan como se configura el campo dentro de la antena en función de la frecuencia de la onda. Debido a las modificaciones en el diseño de las antenas cuando se graba la ranura, la distribución de la densidad de la corriente cambia e induce modificaciones en las frecuencias de resonancia asociada. Estos cambios de frecuencias son evidentes cuando la ranura es un obstáculo significativo a flujo de la densidad de la corriente, para el caso aquí estudiado, cuando la ranura es ortogonal a la dirección de la corriente (en una frecuencia específica). De lo contrario, cuando la ranura es paralela a la dirección de la corriente, no se generan perturbaciones mayores en la frecuencia de resonancia. Lo anterior se entiende como un aumento en la longitud eléctrica de la antena debido a la modifi- cación en su capa radiante, efecto que se considera para un modo o frecuencia de resonancia específica.

\section{AGRADECIMIENTOS}

Los autores agradecen al Instituto Tecnológico Metropolitano por financiar este trabajo a través del proyecto de investigación P15106. Igualmente, la disponibilidad de los semilleros y el grupo de investigación de Automática, Electrónica y Ciencias Computacionales AEyCC

\section{REFERENCIAS}

Balanis, C.A., 2005. Antenna theory: analysis and design. MICROSTRIP ANTENNAS, third edition, John wiley \& sons.

Calabrese, C. \& Marrocco, G., 2008. Meandered-slot antennas for sensor-RFID tags. IEEE Antennas and Wireless Propagation Letters, 7, pp.5-8.

Catano-Ochoa, D. et al., 2016. Performance analysis of a microstrip patch antenna loaded with an array of metamaterial resonators. In 2016 IEEE International Symposium on Antennas and Propagation (APSURSI). IEEE, pp. 281-282.

Fu, Z. \& Yang, F., 2015. A slotted patch antenna integrated with thermal switch for high-sensitivity temperature monitoring. IEEE Antennas and Wireless Propagation Letters, 14, pp.998-1001.

Jang, J.-W. \& Hwang, H.-Y., 2009. An improved band-rejection UWB antenna with resonant patches and a slot. IEEE antennas and wireless propagation letters, 8, pp.299-302.

Joler, M. \& Kucan, J., 2015. Impact of Slot Parameters on the Three Resonant Frequencies of a Rectangular Microstrip Antenna: Study of the impact of the slot length, width, and position. IEEE Antennas and Propagation Magazine, 57(4), pp.48-63.

Khan, Q.U. et al., 2017. Higher Order Modes: A Solution for High Gain, Wide Band Patch Antennas for Different Vehicular Applications. IEEE Transactions on Vehicular Technology, 66(5), pp.3548-3554.

Khidre, A., Yang, F. \& Elsherbeni, A.Z., 2015. A patch antenna with a varactor-loaded slot for reconfigurable dual-band operation. IEEE Transactions on Antennas and Propagation, 63(2), pp.755-760. 
Kim, J., Lee, B. \& Jung, C.W., 2011. Reconfigurable beamsteering antenna using double loops. , 47(7), pp.7-8.

Raval, F., Kosta, Y.P. \& Joshi, H., 2015. Reduced size patch antenna using complementary split ring resonator as defected ground plane. AEU-International Journal of Electronics and Communications, 69(8), pp.11261133.

Reddy, K.S. et al., 2015. Novel Frequency Reconfigurable Slotted Patch Antenna: Design and Analysis. Procedia Materials Science, 10, pp.660-665.

Shivnarayan \& Vishvakarma, B.R., 2005. Analysis of slotloaded rectangular microstrip patch antenna. 84.40 $B a$.

Yang, F. et al., 2012. Reconfigurable sensing antenna: A slotted patch design with temperature sensation. IEEE Antennas and Wireless Propagation Letters, 11, pp.632-635.

Yi, X. et al., 2013. Multi-physics modeling and simulation of a slotted patch antenna for wireless strain sensing. In Structural Health Monitoring 2013: A Roadmap to Intelligent Structures - Proceedings of the 9th International Workshop on Structural Health Monitoring, IWSHM 2013. DEStech Publications, pp. 1857-1864. Available at: http:// www.scopus.com/inward/record.url?eid=2-s2.084945174604\&partnerID=tZOtx3y1.

\section{PARA CITAR ESTE ARTÍCULO TO REFERENCE THIS ARTICLE / PARA CITAR ESTE ARTIGO /}

Ossa Molina, O.; Forero Camen, A.; Espinal Ramírez, C.; ReyesVera, E.; López Giraldo, F. (2017). Efectos en el coeficiente de reflexión de una antena de microcinta rectangular debidos a las modificaciones de posición de una ranura en la capa radiante. Revista EIA, 14(28), julio-diciembre, pp. 85-97. [Online]. Disponible en: https://doi.org/10.24050/reia. v14i28.1143 